\title{
BMJ Open Acute Whiplash Injury Study (AWIS): a protocol for a cluster randomised pilot and feasibility trial of an Active Behavioural Physiotherapy Intervention in an insurance private setting
}

\author{
Taweewat Wiangkham, ${ }^{1}$ Joan Duda, ${ }^{1}$ M Sayeed Haque ${ }^{2}$ Jonathan Price, ${ }^{3}$ \\ Alison Rushton ${ }^{1}$
}

To cite: Wiangkham T, Duda J, Haque MS, et al. Acute Whiplash Injury Study (AWIS): a protocol for a cluster randomised pilot and feasibility trial of an Active Behavioural Physiotherapy Intervention in an insurance private setting. BMJ Open 2016;6: 011336 .

doi:10.1136/bmjopen-2016011336

- Prepublication history and additional material is available. To view please visit the journal (http://dx.doi.org/ 10.1136/bmjopen-2016011336).

Received 28 January 2016 Revised 26 April 2016 Accepted 21 June 2016

CrossMark

For numbered affiliations see end of article.

Correspondence to Taweewat Wiangkham; TXW214@bham.ac.uk

\section{ABSTRACT}

Introduction: Whiplash-associated disorder (WAD) causes substantial social and economic burden internationally. Up to $60 \%$ of patients with WAD progress to chronicity. Research therefore needs to focus on effective management in the acute stage to prevent the development of chronicity. Approximately $93 \%$ of patients are classified as WADII (neck complaint and musculoskeletal sign(s)), and in the UK, most are managed in the private sector. In our recent systematic review, a combination of active and behavioural physiotherapy was identified as potentially effective in the acute stage. An Active Behavioural Physiotherapy Intervention (ABPI) was developed through combining empirical (modified Delphi study) and theoretical (social cognitive theory focusing on self-efficacy) evidence. This pilot and feasibility trial has been designed to inform the design of an adequately powered definitive randomised controlled trial.

Methods and analysis: Two parallel phases. (1) An external pilot and feasibility cluster randomised doubleblind (assessor and participants), parallel two-arm (ABPI vs standard physiotherapy) clinical trial to evaluate procedures and feasibility. Six UK private physiotherapy clinics will be recruited and cluster randomised by a computer-generated randomisation sequence. Sixty participants (30 each arm) will be assessed at recruitment (baseline) and at 3 months postbaseline. The planned primary outcome measure is the neck disability index. (2) An embedded exploratory qualitative study using semistructured indepth interviews ( $\mathrm{n}=3-4$ physiotherapists) and a focus group $(n=6-8$ patients) and entailing the recruitment of purposive samples will explore perceptions of the ABPI. Quantitative data will be analysed descriptively. Qualitative data will be coded and analysed deductively (identify themes) and inductively (identify additional themes).

Ethics and dissemination: This trial is approved by the University of Birmingham Ethics Committee (ERN_15-0542).

Trial registration number: ISRCTN84528320.

\section{Strengths and limitations of this study}

- This is the first pilot and feasibility trial of the Active Behavioural Physiotherapy Intervention $(A B P I)$, which may be a potential useful intervention in preventing patients with acute whiplash-associated disorder (WAD) II progressing to chronicity.

- Employing qualitative and quantitative methods, this trial is designed to evaluate procedures, feasibility and acceptability of the ABPI in managing acute WADII within the UK insurance private sector.

\section{INTRODUCTION}

Whiplash-associated disorder (WAD) describes the variety of symptoms experienced after a whiplash injury, caused by rapid acceleration-deceleration of the head and neck, most commonly following road traffic accidents. ${ }^{1}$ The estimated annual economic cost related to motor vehicle crashes is $\$ 242$ billion in the $\mathrm{USA}^{2}$ and $€ 180$ billion in Europe. ${ }^{3}$ WAD is associated with an increase in healthcare costs, reduced work productivity, lost earning capacity, socioeconomic costs and time contributed by caregivers. ${ }^{4}{ }^{5}$ For example, within the first 2 years after a whiplash injury, employment propensity declined by $20-25 \% .^{4}$ Approximately $60 \%$ of patients with WAD progress to chronicity with up to $30 \%$ experiencing moderate to severe pain and disability, ${ }^{6-8}$ leading to an decrease in quality of life. ${ }^{9}{ }^{10}$ Unfortunately, chronic WAD management is reported to have limited success. ${ }^{6} 71112$ A focus on effective management in the acute stage is therefore required and may be able to prevent patients progressing to chronicity. ${ }^{11} 1314$ 
Over the past decade, an increase in minor cervical spine injuries and related costs after whiplash has been reported from insurance companies. ${ }^{15}$ In the Western World, the cost of insurance claims is considerable, particularly in the UK where a substantial proportion of patients with WAD are managed within the private sector (private physiotherapy clinics) through insurance companies. ${ }^{15-21}$ The UK has also been described as the 'whiplash capital of Europe' by the Association of British Insurers, who estimated that one person in 140 claims for whiplash injury annually. ${ }^{19}$ In the UK, it is estimated that the cost of claims for personal injury have risen from $£ 7$ billion to $£ 14$ billion over the past decade. ${ }^{19}$

WAD has been classified into five grades. ${ }^{1}$ WADII (neck complaint and musculoskeletal sign(s)) describes at least $70 \%$ of patients with whiplash, ${ }^{22} 23$ who are commonly managed by physiotherapists. In the UK, patients with WADII are usually referred to private physiotherapy clinics. ${ }^{19}$ Therefore, evaluating the effectiveness of management of WAD in the private context is important.

Our systematic review of randomised controlled trials (RCTs) ${ }^{13} 14$ evaluating the effectiveness of acute WADII management found that active intervention may be useful for pain reduction medium (95\% CI -17.19 to $-3.23, \mathrm{p}=0.004)$ to long term ( $95 \%$ CI -26.39 to -10.08 , $\mathrm{p}<0.0001)$. Interestingly, the active intervention is also strongly recommended within whiplash management guidelines. ${ }^{24}{ }^{25}$ Second, the review suggested that behavioural intervention may be effective for pain reduction medium term $(95 \%$ CI -15.37 to $-1.55, \mathrm{p}=0.016)$ and improvement of cervical mobility in the coronal $(95 \%$ CI 0.93 to $4.38, \mathrm{p}=0.003)$ and horizontal planes $(95 \% \mathrm{CI}$ 0.43 to $5.46, \mathrm{p}=0.027$ ) short-medium term compared with a standard/control intervention. The combination of active physiotherapy and behavioural interventions, termed 'Active Behavioural Physiotherapy Intervention (ABPI)', may be a useful strategy for acute WADII management to prevent chronicity. ${ }^{14}$

The existing evidence was inadequate to generate an intervention for managing patients with acute WADII. Therefore, the ABPI was developed using empirical (a modified Delphi study by international whiplash researchers, UK private physiotherapists and UK postgraduate musculoskeletal physiotherapy students) (T Wiangkham, J Duda, MS Haque. The development of an Active Behavioural Physiotherapy Intervention (ABPI) for acute Whiplash Associated Disorder (WAD) II management: a modified Delphi study. (Under review) 2016) and theoretical perspectives (social cognitive theory focusing on self-efficacy theory $)^{26}$ in line with the Medical Research Council Framework of Complex Interventions. ${ }^{27}$ Having developed the intervention through a rigorous process, it is now important to explore the feasibility of delivering the intervention in preparation for a future definitive cluster randomised trial. For the UK, the delivery of the ABPI needs to take place in the private setting. ${ }^{28}$

\section{Aims and objectives}

To evaluate procedures, feasibility and acceptability of the ABPI in managing acute WADII within the UK insurance private sector to inform the design and sample size requirements for a future definitive randomised controlled trial.

Primary objectives:

- To evaluate the feasibility of procedures for a cluster randomised controlled trial (eg, randomisation, recruitment, collecting data, trial management and follow-up). ${ }^{29-32}$

- To evaluate the acceptability of the developed intervention. ${ }^{30}$

- To evaluate recruitment rates, refusal rates, compliance of participants in the private sector in the $\mathrm{UK}^{30} 31$

- To evaluate loss of follow-up of participants in the private sector in the UK. ${ }^{30} 32$

Secondary objectives:

- To estimate the required sample size for a clustered definitive trial. ${ }^{30-34}$

- To evaluate the feasibility of data collection for costeffectiveness analysis. ${ }^{30}$

\section{METHODS}

This trial will be conducted according to a predefined protocol (and subsequent deviations will be reported) to minimise potential biases. It follows the Standard Protocol Items: Recommendations for Interventional Trials (SPIRIT) reporting guidelines to ensure sufficient transparency for protocols of clinical trials. ${ }^{35}$ Research methods and reporting are in accordance with the CONSORT 2010 statement: extension to cluster randomised trials ${ }^{36}$ for phase I and COnsolidated criteria for REporting Qualitative research (COREQ): a 32-item checklist for interviews and focus groups ${ }^{37}$ for phase II.

\section{Trial design}

There are two phases to this trial.

\section{Phase I}

An external pilot and feasibility trial of a prospective, cluster randomised double-blind (assessor and participants), parallel two-arm design, comparing ABPI with standard physiotherapy management, will evaluate procedures and feasibility of the ABPI. Six private physiotherapy clinics in the West Midlands, UK will be recruited (figure 1). There are many advantages of cluster randomisation in terms of administrative convenience, ${ }^{38}$ obtaining cooperation of investigators, ethical considerations, ${ }^{38}$ enhancing participant compliance, reducing treatment contamination, ${ }^{28} 363839$ participant blinding $^{36}$ and logistical conveniences. ${ }^{38}$ However, the required sample size in a cluster RCT is larger than an RCT. $^{40}$

Six private physiotherapy clinics will be invited to sign consent forms (cluster-level consent) prior to cluster 


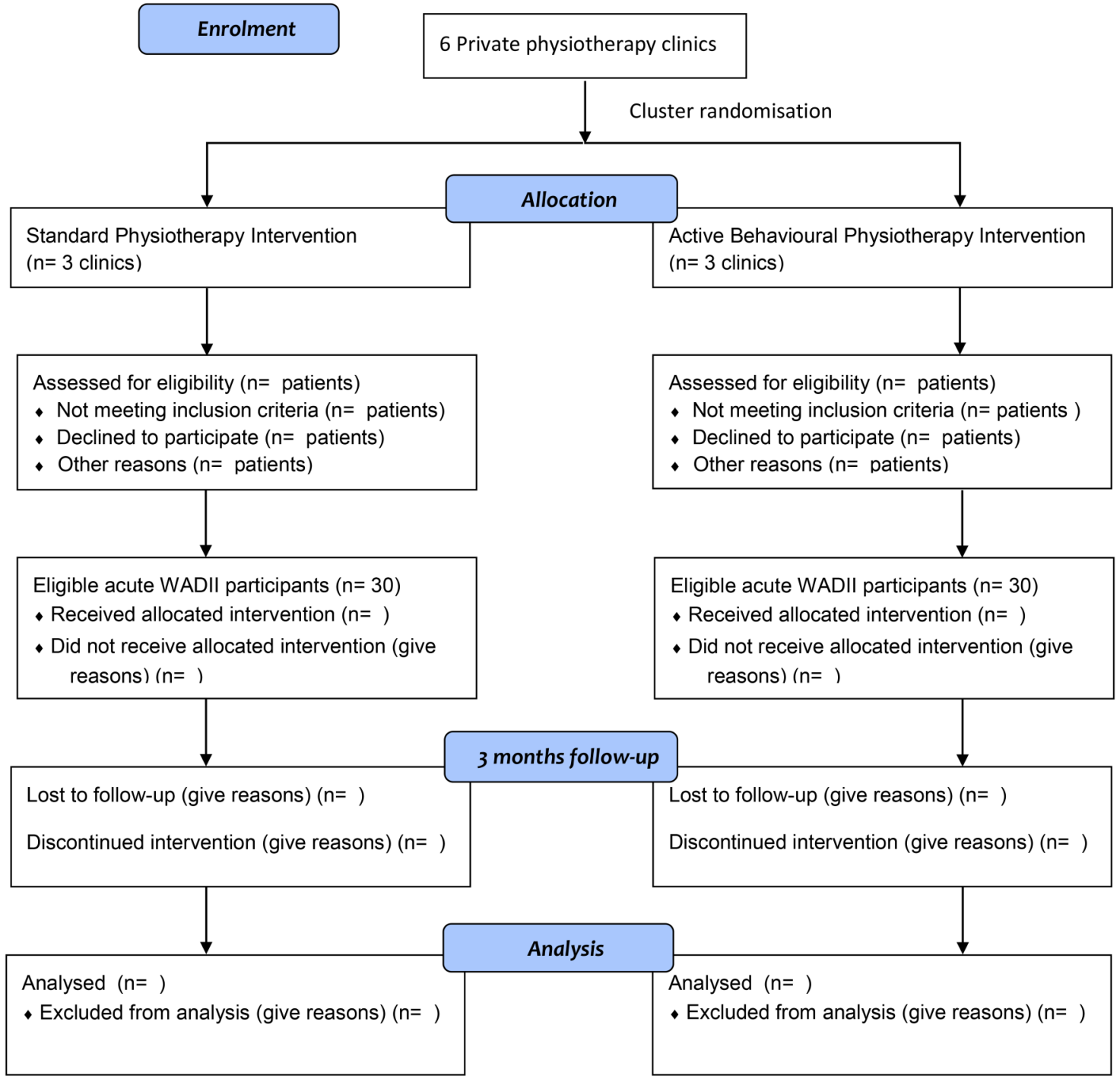

Figure 1 CONSORT flow diagram (adapted from CONSORT 2010). WAD, whiplash-associated disorder.

randomisation. ${ }^{36}$ Following randomisation, consecutive potential participants, referred from an insurance company, will be screened and recruited by a clinical administrator by telephone to book an initial recruitment appointment. The Participant Information Sheet and consent form will be sent via email/post to interested patients to give them the opportunity to read it in advance of the appointment. At the appointment, the recruiting physiotherapist will discuss any issues relating to the trial, confirm eligibility and obtain written consent (individual-level consent). Following informed written consent, participants will be assessed on all outcome measures by a blinded assessor using standardised instruments with established measurement properties. Assessments will be taken at baseline (following recruitment and consent) and at 3 months postbaseline. All outcome assessments will be independent from treatment sessions and treatment clinics to ensure that the assessor is blinded to treatment allocation. The assessor will be a physiotherapist familiar with the outcome measures, and blinded to reduce potential biases. The assessor will not be able to access the booking system and participants' information, whereas participants will not know which intervention arm they will be allocated to ensure that assessor and participants will be blinded. At the end of the 3-month follow-up for each participant, the assessor will be asked 'what intervention the patient had received' and the participants will be asked which intervention arm they had allocated to evaluate the blindness. Two assessment centres central to all clinics will enable convenient attendance for participants. Participants will receive a reminder 2 days prior to the baseline assessment and 3-month follow-up. Providing a reason for participants' withdrawal is voluntary. In the consent form, participants will be asked to confirm if they would like their data removed or kept in the trial, in the situation that they would like to withdraw from the trial (please see online supplementary appendices for the participant information sheet and consent form). 
Phase II

An embedded qualitative study will explore the acceptability of the ABPI for participants and physiotherapists, and to explore how trial procedures and processes worked in practice. ${ }^{41}$ The qualitative study will employ two methods, namely semistructured individual interviews for physiotherapists and a focus group for participants from phase I. Potential participants in either method will be invited via email including an attached participant information sheet and consent form. Prior to performing individual interviews and the focus group, the participants will receive an opportunity to ask questions in order to decide whether they wish to complete the consent form. Demographic characteristics of the participants such as age, gender, occupation and ethnicity will be collected and reported. ${ }^{37}$ Interviewer, moderator and facilitator will be independent from trial interventions, physiotherapy clinics and insurance companies to ensure confidential discussion and avoid potential biases. Transcripts will be returned to participants for any comment and clarification. ${ }^{42}$ The lead researcher (TW) will be trained in individual and focus group interviews to enhance his qualitative skills prior to conducting an interview.

\section{Personal characteristics of research team}

- TW (male): MRes AHR, BSc PT (Hons), Cert GCP, MTPTC. Doctoral Researcher

- AR (female): EdD, MSc, Grad Dip Phys, Cert Ed, Dip TP, FCSP, Health and Care Professions Councils (HCPC), FHEA, FMACP. Senior Lecturer in Physiotherapy and Academic Lead Physiotherapy

- JD (female): PhD, MSc, BA. Professor of Sport and Exercise Psychology

- MSH (male): PhD, MSc, BSc (Hons), FRSS. Senior Lecturer in Medical Statistics

- JP (male): BSc PT. Head of Professional Development

\section{Individual interviews for physiotherapists in the experimental arm}

All physiotherapists who deliver the ABPI (3-4 physiotherapists) will be invited to an individual face-to-face interview by the lead researcher (TW) using a semistructure interview technique. ${ }^{41} 43$ Each interview, which will take 60 min and take place at physiotherapists' clinics, will explore the opinions about attitudes towards and acceptance of the ABPI in managing acute WADII. Furthermore, perceptions of the similarities and differences between standard physiotherapy and the ABPI will be examined. Topic guides for individual interviews will be tested by TW at least two times prior to implementation (table 1). The interview will be noted, audio recorded and transcribed by TW.

\section{Focus group for participants in the experimental arm}

A random sample of $6-8$ participants who received the ABPI will be invited via email to participate in a focus group which is a standard and common procedure for
Table 1 Individual interview theme for the physiotherapists in the experimental arm

\section{Themes Questions}

1

Opinions and attitudes for the new intervention Did you have any obstruction in using the ABPI for treating your patients?

Do you think the ABPI is useful for acute WADII management? Why? Or why not?

What is your opinion regarding the most effective treatment of WADII?

What should be the treatment for acute WADII?

With prompts for detail and elaboration of points

2 Similarities and differences between the standard physiotherapy and the ABPI

What are the similarities and differences between standard physiotherapy and the ABPI? Which intervention do you feel may be more helpful in managing your patients? Why? With prompts for detail and elaboration of points

3 Acceptance of the new intervention Is the ABPI an effective intervention for acute WADII management? Why? Or why not? Do you think the ABPI should be used in managing acute WADII in general? Why? Would you like to change/modify the ABPI? If so how?

With prompts for detail and elaboration of points

ABPI, Active Behavioural Physiotherapy Intervention; WAD, whiplash-associated disorder.

evaluating the acceptability of an intervention. ${ }^{42-44}$ There are several advantages of the focus group including reduced economic compared with one-on-one interviews, and the points that focus groups are conducive to tapping variability in attitudes and opinions due to the interaction facilitated, and provide a comfortable forum for the expression of individual and collective points of view. ${ }^{45} \mathrm{~A}$ reminder email regarding date, time and location of the interview will be sent to the participants 1 day prior to the focus group. The focus group interview/discussion will last for $\sim 1.5$ hours, be held at the University and be led by an expert facilitator (AR) with a moderator (TW) to observe group interaction/dynamics and record main themes of discussion. An important reason for using an expert facilitator is to obtain sufficient quality of the data and to avoid potential biases (eg, consistency bias and dominant respondent bias). ${ }^{45}$ The focus group topic guide will include the intervention that the participants received, the opinions and attitudes of the participants about the intervention, how the participants accepted the intervention and if and how behaviour has changed. Following consent, the focus group will start by agreeing 'ground rules' for the group, including not discussing the content of the group interview outside of the session. The facilitator will start with an introduction for the study and organise questions from general to specific related to interesting topics (table 2). The focus group will be observed, 
Table 2 Focus group theme for the participants in the experimental arm

\begin{tabular}{|c|c|}
\hline Themes & Questions \\
\hline \multirow[t]{6}{*}{1} & Intervention \\
\hline & $\begin{array}{l}\text { What was treatment that you received from your } \\
\text { physiotherapist? }\end{array}$ \\
\hline & How did your physiotherapist approach you? \\
\hline & $\begin{array}{l}\text { What was home programme that you were } \\
\text { recommended to do by your physiotherapist? }\end{array}$ \\
\hline & $\begin{array}{l}\text { What did your physiotherapist suggest for } \\
\text { managing your symptoms? }\end{array}$ \\
\hline & With prompts for detail and elaboration of points \\
\hline \multirow[t]{8}{*}{2} & Opinions and attitudes for the new intervention \\
\hline & Do you think the treatment was useful? Why? \\
\hline & Or why not? \\
\hline & What is your opinion of this treatment for WAD? \\
\hline & What should be the treatment for acute WAD? \\
\hline & $\begin{array}{l}\text { Would you suggest anything in your treatment } \\
\text { be changed or modified? }\end{array}$ \\
\hline & Was there anything missing? \\
\hline & With prompts for detail and elaboration of points \\
\hline \multirow[t]{7}{*}{3} & Acceptance of the new intervention \\
\hline & How did you feel after receiving the treatment? \\
\hline & Do you accept the treatment that your \\
\hline & physiotherapist gave to you? Why? \\
\hline & $\begin{array}{l}\text { What is/are the benefit(s) of the treatment that } \\
\text { you received from your physiotherapist? }\end{array}$ \\
\hline & $\begin{array}{l}\text { Do you think the treatment should be used for } \\
\text { acute WADII management? Why? Or why not? }\end{array}$ \\
\hline & With prompts for detail and elaboration of points \\
\hline \multirow[t]{7}{*}{4} & Behavioural changes \\
\hline & What differences, in your lifestyle, did you \\
\hline & notice after receiving the treatment? \\
\hline & After going through this treatment, have you \\
\hline & committed to adopting a more healthy lifestyle? \\
\hline & If yes, how? If not, why not? \\
\hline & With prompts for detail and elaboration of points \\
\hline
\end{tabular}

noted, audio recorded and transcribed by TW. The participants' names will not be linked to any information in the reporting of findings from the group discussion, and findings will be reported for the whole group rather than for individual participants. After the focus group, the moderator and facilitator will discuss the main findings and unexpected outcomes. ${ }^{42}$

\section{Participants}

Participants will be recruited from six UK private physiotherapy clinics. Demographic characteristics, including age, gender, accident history, present drugs, information regarding WAD symptoms, will be taken by the blinded assessor at the baseline assessment. The participants in this trial can normally claim all expenditures regarding their treatment sessions from their insurance company. The trial will pay for the participants' journeys at baseline and 3-month follow-up that are additional contact points.
Eligibility criteria for clusters: private clinics in the West Midlands, UK. Preliminary data have identified that each clinic has at least two patients presenting with acute WADII each month.

Inclusion criteria: Participants aged $18-70$ years old, presenting with WAD grade II (neck complaint and musculoskeletal $\operatorname{sign}(\mathrm{s}))^{1}$ from a road traffic accident within the previous 4 weeks. $7142546-48$

Exclusion criteria: Signs and symptoms of upper cervical instability ${ }^{49}$ or cervical artery dysfunction, ${ }^{50}$ suspected serious spinal pathology, open wounds, active inflammatory arthritis, tumours, infection of the skin and soft tissue, bleeding disorders or using anticoagulant medication, ${ }^{49}$ any current or previous treatment from any other third party or presenting with any serious injuries from other areas of the body resulting from the accident, history of cervical surgery, ${ }^{51}$ previously symptomatic degenerative diseases of the cervical spine within 6 months before the road traffic accident, ${ }^{52}$ previous history of whiplash or other neck pain, ${ }^{47}$ alcohol abuse, ${ }^{52} 53$ dementia, ${ }^{52}{ }^{53}$ serious mental diseases, ${ }^{52} 53$ psychiatric diseases $^{54} 55$ and/or non-English speaking and reading.

\section{Interventions}

Interventions are described based on Template for Intervention Description and Replication (TIDieR). ${ }^{56}$ Participants in both intervention arms will attend face-to-face physiotherapy sessions lasting for up to 30 min once a week in a private physiotherapy clinic. The number of treatment sessions will vary between 6 and 8 sessions based on the individual physiotherapist's assessment. All physiotherapists in both intervention arms will have a minimum of a Bachelor Degree in Physiotherapy with 2 years of postregistration experience, and will be registered with the HCPC. To evaluate fidelity of the ABPI, a summary of treatment sessions will be systematically collected and sessions will be randomly observed by the lead researcher (TW). This will enable monitoring and feedback regarding the intervention to the treating physiotherapist.

\section{Standard physiotherapy intervention}

Patients will be managed according to current practice reflecting the recommendations provided in the clinical whiplash guidelines. ${ }^{24} 2548$ Physiotherapy interventions such as reassurance, education, manual therapy, exercise therapy and physical agents, including a home programme of exercises, are part of management depending on the individual physiotherapist's clinical reasoning for the individual patient. The treating physiotherapists select appropriate interventions based on examination findings and clinical reasoning. ${ }^{50}$

\section{Active Behavioural Physiotherapy Intervention}

The specific detail of this intervention including the underlying principles (eg, return to normal function/movement as soon as possible, encourage self-management, and 
reduce fear avoidance and anxiety) and the specific treatment components in physical (eg, exercise programmes for stability and mobility) and psychological (eg, cognitivebehavioural therapy, whiplash education, advice to act as usual, reassurance, self-management and postural control and education) aspects were developed by international research and local clinical whiplash experts through a modified Delphi method ( $\mathrm{T}$ Wiangkham, et al Under review). By consideration of empirical and theoretical perspectives in line with the Medical Research Council Framework of Complex Interventions, ${ }^{27}$ social cognitive theory (with a particular focus on self-efficacy enhancement) will be used to underpin the ABPI to manage the patient with acute WADII. ${ }^{26}{ }^{57}$ Past research has found selfefficacy to correlate with quality of life and health status in physical (eg, pain and physical functions) and psychological (eg, anxiety and depression) perspectives in a rehabilitation context. ${ }^{58} 59$

The ABPI for acute WADII management consists of four phases in terms of the promotion of understanding, maturity, stamina and coping (figure 2). Table 3 presents a summary of the ABPI for acute WADII management. The number of treatment session in each phase will vary depending on individual patients' conditions based on physiotherapist's justification. The recommendation is $\sim 1-3$ visits in each phase.

Training of physiotherapists in the experimental arm to deliver the ABPI will be delivered in advance of data collection. The training will consist of a group tutorial and workshop followed by individual training sessions to construct the concept of how to design the intervention and how to manage patients with WADII using the ABPI programme based on the findings of the subjective and objective examinations, and evidence informed clinical reasoning. ${ }^{50}$ The physiotherapists will have 4 weeks to practise their skills embedded in the ABPI in managing patients with acute WADII prior to participants' recruitment. They will be randomly observed by the lead researcher (TW) every week before starting participant recruitment and every month during data collection. Feedback will be provided throughout the trial.

\section{Outcomes}

\section{Planned primary outcome measure}

The neck disability index (NDI) is a patient-reported outcome measure and a valid, reliable and responsive tool in assessing pain and disability of neck in acute and chronic conditions. ${ }^{60-63}$ The NDI is a self-administered questionnaire that includes 10 sections focusing on functional activities such as pain intensity, personal care, lifting, reading, headache, concentration, work, driving, sleeping and recreation. ${ }^{60}$ Each section is scored $0-5$, with 5 representing the greatest disability. The sum is calculated and converted into a percentage to indicate the participant's perceived level of disability. ${ }^{60}$ The NDI is a robust predictor of outcome for acute $\mathrm{WAD}^{64}$ and recommended to monitor patients with whiplash by several clinical guidelines, including NHS Library, New South Wales Motor Accidents Authority, British Columbia Physiotherapy Association, Royal Dutch Society for Physical Therapy and the South Australian Centre for Trauma and Injury. ${ }^{25} 4862$ Consequently, several previous whiplash intervention trials have used the NDI as the primary outcome. ${ }^{47} 65$

\section{Planned secondary outcome measures \\ Visual analogue scale for pain intensity}

The most common symptom in patients with whiplash is pain. ${ }^{18}$ Pain will be measured using a $0 \mathrm{~mm}$ (no pain) to $100 \mathrm{~mm}$ (worst possible pain) visual analogue scale (VAS) ${ }^{66}$ which is a simple and preferred tool for assessing pain intensity, with high validity and reliability in evaluating acute pain. ${ }^{67-69}$ The identification of initial pain intensity using the VAS has been found to be an important prognostic factor in predicting poor recovery in patients with acute whiplash. ${ }^{64} 70$

\section{Cervical range of motion}

Decreasing cervical mobility is a common finding in patients with WADII. ${ }^{71}$ Cervical range of motion (CROM) is highly sensitive and can be specifically tested for discrimination between asymptomatic and symptomatic whiplash ${ }^{72}$ and for handicap prediction of acute whiplash injury. ${ }^{73}$ In this trial, the CROM will be measured by the cervical range of motion device. ${ }^{74}$ The cervical range of motion device is a highly valid and reliable device in measuring CROM and is attached to the head. ${ }^{75-77}$ The participant sits on a comfortable chair with hips and knees flexed to $90^{\circ}$. CROM measurements are recorded three times in each movement direction. The mean of the three measurements will be used for data analysis.
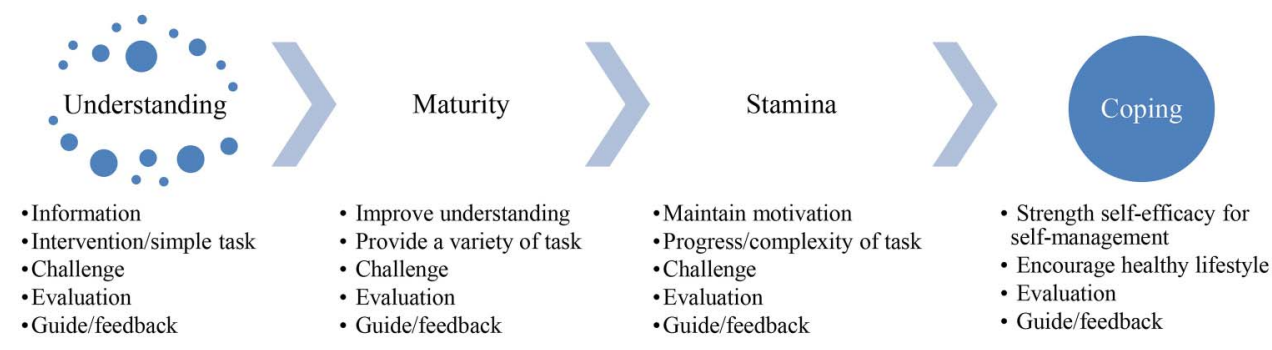

Figure 2 Active Behavioural Physiotherapy Intervention for acute whiplash-associated disorder II management. 
Table 3 ABPI for acute WADII management

\begin{tabular}{lll}
\hline Phases & Strategies & Goals \\
\hline 1. & Information & Increase self-efficacy to reduce \\
Understanding & Intervention/simple & psychological stress and \\
& task & confidence in exercises by \\
& Challenge & education. \\
& Evaluation & Initiate gentle exercise for \\
& maintain/improve neck stability \\
& & and mobility. \\
& & Promote self-management \\
& psychological and physical \\
& management.
\end{tabular}

2. Maturity

3. Stamina

\author{
- Improve \\ understanding \\ - Provide a variety of \\ task \\ - Challenge \\ - Evaluation \\ - Guide/feedback
}

- Increase self-efficacy to reduce psychological stress and confidence in exercises.

- Exercises for neck and shoulder stability and mobility.

- Promote self-management for pain and physical functions.

\section{Interventions}

- Increase self-efficacy using physiotherapist provided verbal persuasion with the aim of reducing psychological stress and confidence in exercises by whiplash education.

- Initiate gentle exercises and home programmes including challenge for neck stability and mobility exercises (eg, isometric neck exercises, chin in and active CROM with pain free).

- Promote self-management to include psychological (eg, stress management and relaxation techniques) and physical (eg, pain reduction and physical functions) aspects.

- Other physiotherapy programmes based on clinical reasoning.

- Increase self-efficacy (reduce psychological stress and improve confidence in performing exercises)

- Performance accomplishment (eg, relieve pain and increase CROM);

- Verbal persuasion (eg, further whiplash education/feedback when patients need, continue exercises with challenge);

- Increase emotional stages with good relationship.

- Exercises+home programmes including challenge for neck and shoulder stability and mobility exercise (eg, resisted neck and shoulder, and AROM exercises).

- Promote self-management for pain and physical functions (psychological management when patients need).

- Other physiotherapy programmes based on clinical reasoning.

- Increase/maintain self-efficacy for self-management and exercises

- Performance accomplishment (eg, relieve pain, increase CROM, improve physical functions);

- Verbal persuasion (eg, guide/ feedback, continue exercises with challenge);

- Increase/maintain emotional stages with good relationship.

- Progressive exercises+home programmes including challenge for strengthening and ROM exercises.

- Promote self-management for physical functions.

- Other physiotherapy programmes based on clinical reasoning. 
Table 3 Continued

\begin{tabular}{lll}
\hline Phases & Strategies & Goals \\
\hline 4. Coping & Strength & Maintain/increase self-efficacy \\
& self-efficacy for & for self-management and \\
self-management & exercises. \\
& Encourage healthy & Promote self-management for \\
lifestyle & physical functions. \\
& Evaluation & Facilitate long-term goal for \\
& Guide/feedback & healthy lifestyle.
\end{tabular}

Interventions

- Maintain/increase self-efficacy

- Performance accomplishment (eg, physical functions);

- Verbal persuasion (eg, guide/ feedback, continue exercises with challenge to be a healthy lifestyle person);

- Increase/maintain emotional stages with good relationship.

- Home programmes for strengthening and ROM exercises.

- Promote self-management for physical functions.

- Facilitate the adoption/maintenance of a healthy lifestyle.

- Other physiotherapy programmes based on clinical reasoning.

ABPI, Active Behavioural Physiotherapy Intervention; CROM, cervical range of motion; WAD, whiplash-associated disorder.

\section{Pressure pain threshold}

Pressure pain threshold (PPT) is measured using minimal pressure force to identify the threshold of stimulating pain. ${ }^{78}$ Patients with whiplash frequently report regarding central hyperexcitability in acute $(<1 \text { month })^{79-81}$ and chronic stages. ${ }^{82}$ The investigation of PPT at remote pain-free muscles suggests that a component of hypersensitivity in patients with whiplash may come from central sensitisation. ${ }^{83}$ A digital pressure algometer is a highly valid and reliable instrument, used to detect sensitivity of symptomatic areas and distal painfree areas. ${ }^{84} 85$ The speed of applied force is $30 \mathrm{kPa} / \mathrm{s}^{81}$ The participants are required to press a button when their sensation changes from pressure to perceived pain. ${ }^{81}$ PPT will be assessed at the insertion of the levator scapulae ${ }^{81}$ and the upper one-third of the tibialis anterior muscle ${ }^{85}$ on both sides, three times each side, with an interval of 1 min between each test. ${ }^{86}{ }^{87}$ The mean of the three measurements will be used for data analysis. The starting position of the assessment is comfortable upright sitting with hip and knee flex $90^{\circ}$ for the levator scapulae and supine lying with the knee of an assessed side flex $90^{\circ}$ for the tibialis anterior.

\section{Impact of events scale}

The impact of event scale (IES) is a valid and reliable 15-item questionnaire assessing current stress and indicating the symptoms of post-traumatic stress ${ }^{88-90}$ that may contribute to a high risk of persistent symptoms. ${ }^{54}$ 91-93 The IES is recommended by some clinical whiplash guidelines to monitor whiplash management. ${ }^{25} 48$

\section{Fear-Avoidance Beliefs Questionnaire}

The physical disability of patients with WAD can be influenced by fear-avoidance beliefs and associated behaviours following whiplash injury. ${ }^{94-96}$ Patients with dysfunctional illness beliefs need to have these addressed to prevent chronicity. ${ }^{97}$ The Fear-Avoidance Beliefs Questionnaire is a 16-item valid and reliable tool administered to patients with neck pain, ${ }^{98}$ to assess their perceptions of the impact of physical activity and work on their levels of pain and disability.

\section{Assessment of outcome}

Masked assessment of outcomes will take place at baseline and at 3 months postbaseline. After 3 months, the patients with whiplash who continue with symptoms and problems are defined as chronic. ${ }^{725}$ In the future definitive trial, the primary end point will be 3 months and the number of recovered patients with WADII within 3 months will be evaluated. Longer term follow-up is also planned to 1 year. Participants who do not attend the 3-month follow-up assessment will be contacted by telephone and asked if they would like to make a new appointment. If they cannot make a new appointment, the researcher will ask them to complete the NDI via telephone interview, which has established reliability and validity. $^{99}$

\section{Feasibility of cost-effectiveness analysis}

Direct and indirect medical costs will be collected to assess the feasibility of data collection for the planned cost-effectiveness analysis in the definitive trial. Participants will receive a diary pocket book to record any activities related to whiplash management such as using medication, consulting other health professionals; along with any costs they incur, days of sick leave, benefits claimed that relate to whiplash management. In the first page of the diary pocket book, general information on participants (eg, postcode, work status and income) will be collected. Costs related to physiotherapy management will be collected from the physiotherapy clinics. 
Training costs of physiotherapists in the experimental ABPI arm will also be recorded. The quality-adjusted life years will be estimated using the advocated EuroQol-5 Dimensions. ${ }^{100}$

\section{Sample size}

As this is a pilot and feasibility trial, a power calculation is not required. ${ }^{30}$ Although establishing targeted sample sizes for pilot/feasibility trials is controversial, 60 participants (30 per arm) will be recruited to provide sufficient power of parameters for designing an adequate power randomised controlled trial. ${ }^{101}$ Data from the physiotherapy clinics provided evidence of 18 eligible participants available per month across the six private physiotherapy clinics. The recruitment rate of this trial will be considered adequate if it is at least $50 \%$ of eligible participants are recruited. Based on this estimate, the trial may take 6-7 months for participant recruitment with 3-month follow-up.

\section{Randomisation}

To minimise selection bias at the cluster level, a computer-generated randomisation programme will be used by the lead researcher (TW) to randomise six private physiotherapy clinics into two groups: standard physiotherapy intervention ( $\mathrm{n}=3$ clinics) and ABPI $(\mathrm{n}=3$ clinics). Allocation will be concealed prior to assignment. Only TW will be involved in this process. Cluster randomisation will be implemented before participants are recruited (see figure 1 for CONSORT flow diagram).

\section{Data analysis}

Phase I

Data will be analysed and summarised based on quantitative synthesis using a prespecified protocol to evaluate eligible, recruitment and follow-up rates. Quantitative data will be analysed using IBM SPSS V.22. Descriptive statistics will assess the feasibility of the ABPI for acute WADII management to inform the design of the future definitive trial (table 4). The participants who receive other treatments from the initial randomised treatment allocation will not be disregarded in the trial and their data will be included in intention-to-treat analyses. The planned primary end point of this trial is evaluation of the NDI at 3-month follow-up. Evaluation of the drop-out rate of participants will be a criterion to confirm the primary end point. Upon completion of the pilot and feasibility trial, the following possible decisions will be considered by evaluating the feasibility criteria (table 4) for conducting the definitive trial: ${ }^{31}$

Table 4 Feasibility assessment criteria

\section{Objectives}

To evaluate the feasibility of procedures (eg, randomisation, recruitment, collecting data, management, follow-up) ${ }^{29-32}$

To investigate the acceptability of the developed intervention ${ }^{30}$

To evaluate recruitment rates, refusal rates, retention, compliance of participants in the private sector in the $\mathrm{UK}^{30} 31$

To evaluate dropout rates of participants in the private sector in the $\mathrm{UK}^{30} 32$

To estimate the required sample for a definitive trial ${ }^{30-34}$

To evaluate the feasibility of data collection for cost-effectiveness analysis ${ }^{30}$

\section{Criteria of success}

The trial will be considered feasible if this trial can be run smoothly without serious problems or obstructions which are able to stop the study. ${ }^{29} 31$

The trial will be considered feasible if the physiotherapists and the participants find the developed intervention acceptable.

The trial will be considered feasible if

- $\geq 50 \%$ of eligible participants can be recruited;

- at least three participants a week per intervention arm can be recruited;

- $\geq 80 \%$ of all recruited participants complete the follow-up at 3 months.

The trial will be considered feasible if $\leq 20 \%$ of all recruited participants dropout.

The trial will be considered feasible if the sample size for a cluster RCT is feasible to achieve based on recruitment data. The trial will be considered feasible if the following components of the cost-effective analysis can be collected with minimal missing data.

- General information (eg, current work status and salary).

- Direct medical costs

- Medical costs (eg, physiotherapy, general practice and complementary medicine);

- Resource uses (eg, diagnosis tests).

- Indirect medical costs

- Participant journey costs;

- Training costs for physiotherapists in the experimental arm.

ABPI, Active Behavioural Physiotherapy Intervention; RCT, randomised controlled trial; WAD, whiplash-associated disorder. 
- Stop if the main trial is not possible or valuable.

- Continue but modify the protocol if the main trial is possible and valuable.

- Continue without modifications but monitor closely if the main trial is possible and valuable with close monitoring.

- Continue without modifications if the main trial is possible and valuable.

The intracluster correlation coefficient will also be calculated to prepare information for sample size calculation within a clustered definitive trial.

\section{Phase II}

Qualitative data of individual interviews and focus group will be coded and by the lead researcher (TW). QRS NVivo 10 will be employed to identify themes regarding the acceptability of the ABPI to physiotherapists and participants, and how trial procedures and processes worked in practice. ${ }^{102} 103$ In the focus group, a key aim of the analysis is to identify any emerging group consensus regarding attitudes towards and experiences of the ABPI. ${ }^{104}$ The participants' name will not be linked to any information in the reporting of findings from the group discussion, and findings will be reported for the whole group rather than for individual participants. The data will be analysed deductively (to identify themes) and inductively (to identify additional themes). ${ }^{44} 105$ The analysis and findings emanating from the qualitative data will be discussed with the research team at each stage (TW, AR, JD and MSH). The mapping and interpretation of the data will be used to explore and explain relevant patterns. The interpretation of qualitative data will be carried out in parallel to the quantitative findings.

\section{Trial management and monitoring}

The trial will be managed by the Trial Management Group consisting of TW, AR, JD and MSH. The trial combines the Trial Steering Committee and the Data Monitoring Committee functions in line with the nature of the trial, into the Acute Whiplash Injury Study (AWIS) Steering Group, consisting of TW, AR, MSH, JP, a WADII patient, an external member and an independent chair. The committee will meet at the start of recruitment, after 3 months of recruitment and at completion of data collection. The lead researcher (TW) has qualified for Good Clinical Practice (certificate number: 33951-36-41796).

\section{Adverse events}

Adverse events in this trial are considered as low risk. First, WADII (neck complaint and musculoskeletal sign (s)) is not normally a cause of serious adverse events. ${ }^{11}{ }^{65}$ Second, the ABPI and standard physiotherapy intervention are conservative treatments without existing reporting of serious adverse events. ${ }^{11}{ }^{65}$ As a result, patients are unlikely to receive any serious harm from either intervention. Generally, only minor adverse events are likely to occur after the physiotherapy intervention. The most common adverse events for the physiotherapy intervention are muscle soreness that commonly recovers within $1-2$ days. ${ }^{106}$

\section{Serious adverse events}

This trial will have a very low risk of serious adverse events in terms of patient pathology, treatment nature and treatment management. Participants will be evaluated by a physiotherapist prior to seeking consent to ensure that the participants are classified as WADII (presented only musculoskeletal sign(s) without any neurological sign(s)) to meet the eligibility criteria, thereby excluding patients with high severity. All physiotherapists in this trial manage their patients based on the International Federation of Orthopaedic Manipulative Physical Therapists cervical framework ${ }^{50}$ that provides a framework for clinical reasoning to avoid risk of any adverse events regarding the vascularity and instability of the neck from physical therapy intervention. However, progressive symptoms within 3 days and admitted in the hospital due to whiplash problems will be reported for serious adverse events. If any serious adverse events occur, the patients will be able to continue with the trial when their symptoms are resolved.

\section{Procedures for reporting adverse and serious adverse events}

An adverse event reporting form will be provided to all clinics. If a participant experiences any unpleasant symptoms, they will be asked to report them to their physiotherapist. The physiotherapist will report any event to the researcher (TW) within 24 hours. The researcher will report to the AWIS steering committee within 24 hours to enable analysis of the event and any required action. Although this trial may have low risk of adverse events, any sign(s) and/or symptom(s), which would cause life-threatening situations, inpatient hospitalisation and significant disability (eg, unable to work), may occur. Any unexpected serious adverse events will be immediately reported with a written form and verbal contact by physiotherapists to the researcher (TW). Then, the researcher will report to the AWIS steering committee immediately.

\section{Research governance}

The trial will maintain research governance by using the principles of the Research Governance Framework for Health and Social Care.

\section{Data management}

All information collected on and from the participants will be kept safely from any third party to maintain the participants' privacy. All collected documents will be stored in a secure place. All electronic data will also be confidentially stored in a password protected computer during the trial. Data can only be accessed by members 
of the research team. The findings will be submitted for publication to medical journals and presented at conferences and local seminars. The trial will only be published in a completely unattributable format or at an aggregate level in order to ensure that no participant can be identified. After completing the trial, all data will be destroyed after being kept for 10 years at the School of Sport, Exercise and Rehabilitation Sciences, University of Birmingham.

\section{R\&D considerations}

NHS ethical approval and R\&D approval is not required as the trial sites are outside of the UK National Health Service. The insurance/private clinics do not require any other regulatory approval. Support for the trial is in place by the private clinics and the insurance companies.

\section{DISCUSSION}

Before designing and conducting an adequate powered, high-quality cluster RCT examining the ABPI for managing acute WADII, this pilot and feasibility trial is required. If the pilot and feasibility trial is successful (ie, the ABPI is found to be feasible), a future definitive trial will be implemented to compare the effectiveness of the ABPI and standard physiotherapy intervention, inclusive of cost-effectiveness analysis. If it is demonstrated that the ABPI is effective in managing patients with acute WADII, the ABPI will be a valuable intervention to prevent patients with acute WADII progressing to chronicity.

\section{Author affiliations}

${ }^{1}$ School of Sport, Exercise and Rehabilitation Sciences, College of Life and Environmental Sciences, University of Birmingham, Birmingham, UK ${ }^{2}$ Institute of Clinical Sciences, College of Medical and Dental Sciences, University of Birmingham, Birmingham, UK

${ }^{3}$ Physio 1st, Birmingham, UK

Acknowledgements The study is not funded from any sources. However, the lead author (TW) thanks the Royal Thai Government and Naresuan University for the scholarship to support his PhD work. Furthermore, The authors thank the University of Birmingham for the sponsorship.

Contributors TW, AR, JD, MSH and JP have made substantial intellectual contributions to the trial. All authors have read and approved the final manuscript.

\section{Competing interests None declared.}

Ethics approval the University of Birminghamâ€s Ethics Committee

Provenance and peer review Not commissioned; externally peer reviewed.

Open Access This is an Open Access article distributed in accordance with the Creative Commons Attribution Non Commercial (CC BY-NC 4.0) license, which permits others to distribute, remix, adapt, build upon this work noncommercially, and license their derivative works on different terms, provided the original work is properly cited and the use is non-commercial. See: http:// creativecommons.org/licenses/by-nc/4.0/

\section{REFERENCES}

1. Spitzer WO, Skovron ML, Salmi LR, et al. Scientific monograph of the Quebec Task Force on Whiplash-Associated Disorders: redefining 'whiplash' and its management. Spine (Phila Pa 1976) 1995;20(Suppl 8):1s-73S.
2. Blincoe L, Miller TR, Zaloshnja E, et al. The economic and societal impact of motor vehicle crashes, 2010 (revised), 2015.

3. Elvik MR, Ortenwall MP, Mackay MM, et al. Social and economic consequences of road traffic injury in Europe. Brussels, Belgium 2007.

4. Leth-Petersen S, Rotger GP. Long-term labour-market performance of whiplash claimants. J Health Econ 2009;28:996-1011.

5. Jennum P, Kjellberg J, Ibsen R, et al. Health, social, and economic consequences of neck injuries: a controlled national study evaluating societal effects on patients and their partners. Spine (Phila Pa 1976) 2013;38:449-57.

6. Merrick $D$, Stålnacke BM. Five years post whiplash injury: symptoms and psychological factors in recovered versus non-recovered. BMC Res Notes 2010;3:190.

7. Sterling M. Physiotherapy management of whiplash-associated disorders (WAD). J Physiother 2014;60:5-12.

8. Jull GA, Sterling M, Curatolo M, et al. Toward lessening the rate of transition of acute whiplash to a chronic disorder. Spine (Phila Pa 1976) 2011;36(Suppl 25):S173-4

9. Börsbo B, Peolsson M, Gerdle B. Catastrophizing, depression, and

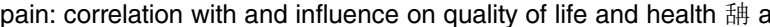
study of chronic whiplash-associated disorders. J Rehabil Med 2008;40:562-9.

10. Börsbo B, Peolsson M, Gerdle B. The complex interplay between pain intensity, depression, anxiety and catastrophising with respect to quality of life and disability. Disabil Rehabil 2009;31:1605-13.

11. Michaleff ZA, Maher CG, Lin CW, et al. Comprehensive physiotherapy exercise programme or advice for chronic whiplash (PROMISE): a pragmatic randomised controlled trial. Lancet 2014;384:133--41.

12. Sterling M, Vicenzino B, Souvlis T, et al. Dry-needling and exercise for chronic whiplash-associated disorders: a randomized single-blind placebo-controlled trial. Pain 2015;156:635-43.

13. Wiangkham T, Duda J, Haque MS, et al. Effectiveness of conservative treatment in acute whiplash associated disorder (WAD) II: a systematic review and meta-analysis of randomised controlled trials. Physiotherapy 2015;101(Suppl 1):e1623-e24.

14. Wiangkham T, Duda J, Haque $\mathrm{S}$, et al. The effectiveness of conservative management for acute whiplash associated disorder (WAD) II: a systematic review and meta-analysis of randomised controlled rrials. PLOS ONE 2015;10:e0133415.

15. Buitenhuis J, de Jong PJ, Jaspers JP, et al. Work disability after whiplash: a prospective cohort study. Spine (Phila Pa 1976) 2009;34:262-7.

16. Côté P, Hogg-Johnson S, Cassidy JD, et al. Early aggressive care and delayed recovery from whiplash: isolated finding or reproducible result? Arthritis Rheum 2007;57:861-8.

17. Chappuis G, Soltermann B. Number and cost of claims linked to minor cervical trauma in Europe: results from the comparative study by CEA, AREDOC and CEREDOC. Eur Spine J 2008;17:1350-7.

18. Holm LW, Carroll LJ, Cassidy JD, et al. The burden and determinants of neck pain in whiplash-associated disorders after traffic collisions: results of the Bone and Joint Decade 2000-2010 Task Force on Neck Pain and Its Associated Disorders. Spine (Phila Pa 1976) 2008;33(Suppl 4):S52-9.

19. Mooney $\mathrm{H}$. Insurance companies are reeling from the number of claims being made by people who say they have whiplash injuries. 2012. http://www.csp.org.uk/frontline/article/what $\%$ E2\%80\% 99s-driving-rise-whiplash-injuries (accessed Oct 2013).

20. Hyde D. Why Britain is the whiplash capital of Europe. The Telegraph. United Kingdom, 2013.

21. FSCO. Submission to the superintendent, financial services, FSCO: submitted for the review of part VI of the insurance act. Canada: Financial Services Commission of Ontario, 2014.

22. Sterling M. A proposed new classification system for whiplash associated disorders-implications for assessment and management. Man Ther 2004;9:60-70.

23. Williamson E, Williams MA, Gates $\mathrm{S}$, et al. Risk factors for chronic disability in a cohort of patients with acute whiplash associated disorders seeking physiotherapy treatment for persisting symptoms. Physiotherapy 2015;101:34-43.

24. Moore A, Jackson A, Jordan J, et al. Clinical guidelines for the physiotherapy management of whiplash associated disorder. London: Chartered Society of Physiotherapy, 2005.

25. TRACsa. Clinical guidelines for best practice management of acute and chronic whiplash associated disorders: clinical resource guide. Adelaide: TRACsa: Trauma and Injury Rocovery, 2008.

26. Bandura A. Self-efficacy: toward a unifying theory of behavioral change. Psychol Rev 1977;84:191-215.

27. Craig P, Dieppe P, Macintyre S, et al. Developing and evaluating complex interventions: the new Medical Research Council guidance. BMJ 2008;337:a1655. 
28. Siebers AG, Klinkhamer PJ, Grefte JM, et al. Comparison of liquid-based cytology with conventional cytology for detection of cervical cancer precursors: a randomized controlled trial. JAMA 2009;302:1757-64.

29. Lancaster GA, Dodd S, Williamson PR. Design and analysis of pilot studies: recommendations for good practice. J Eval Clin Pract 2004:10:307-12.

30. Arain $\mathrm{M}, \mathrm{Campbell} \mathrm{MJ}$, Cooper $\mathrm{CL}$, et al. What is a pilot or feasibility study? A review of current practice and editorial policy. BMC Med Res Methodol 2010;10:67.

31. Thabane L, Ma J, Chu R, et al. A tutorial on pilot studies: the what, why and how. BMC Med Res Methodol 2010;10:1.

32. Whitehead AL, Sully BG, Campbell MJ. Pilot and feasibility studies: is there a difference from each other and from a randomised controlled trial? Contemp Clin Trials 2014;38:130-3.

33. Gould AL. Planning and revising the sample size for a trial. Stat Med 1995;14:1039-51; discussion 53-5.

34. Coffey CS, Muller KE. Properties of internal pilots with the univariate approach to repeated measures. Stat Med 2003;22:2469-85.

35. Chan AW, Tetzlaff JM, Gotzsche PC, et al. SPIRIT 2013 explanation and elaboration: guidance for protocols of clinical trials. BMJ 2013;346:e7586

36. Campbell MK, Piaggio G, Elbourne DR, et al. Consort 2010 statement: extension to cluster randomised trials. BMJ 2012;345: e5661.

37. Tong A, Sainsbury P, Craig J. Consolidated criteria for reporting qualitative research (COREQ): a 32-item checklist for interviews and focus groups. Int J Qual Health Care 2007;19:349-57.

38. Edwards SJ, Braunholtz DA, Lilford RJ, et al. Ethical issues in the design and conduct of cluster randomised controlled trials. BMJ 1999;318:1407-9.

39. Wyatt JC, Paterson-Brown S, Johanson R, et al. Randomised trial of educational visits to enhance use of systematic reviews in 25 obstetric units. BMJ 1998;317:1041-6.

40. Teerenstra S, Eldridge S, Graff M, et al. A simple sample size formula for analysis of covariance in cluster randomized trials. Stat Med 2012;31:2169-78

41. Graham HL, Birchwood M, Griffith E, et al. A pilot study to assess the feasibility and impact of a brief motivational intervention on problem drug and alcohol use in adult mental health inpatient units: study protocol for a randomized controlled trial. Trials 2014;15:308

42. De Cocker K, Veldeman C, De Bacquer D, et al. Acceptability and feasibility of potential intervention strategies for influencing sedentary time at work: focus group interviews in executives and employees. Int J Behav Nutr Phys Act 2015;12:22.

43. Stow R, Rushton A, Ives N, et al. A cluster randomised feasibility trial evaluating six-month nutritional interventions in the treatment of malnutrition in care home-dwelling adults: recruitment, data collection and protocol. Pilot Feasibility Stud 2015;1:3

44. Ayala GX, Elder JP. Qualitative methods to ensure acceptability of behavioral and social interventions to the target population. $J$ Public Health Dent 2011;71(Suppl 1):S69-79.

45. Sim J. Collecting and analysing qualitative data: issues raised by the focus group. J Adv Nurs 1998;28:345-52.

46. Sterling M, Kenardy J. The relationship between sensory and sympathetic nervous system changes and posttraumatic stress reaction following whiplash injury-a prospective study. $J$ Psychosom Res 2006;60:387-93.

47. Jull G, Kenardy J, Hendrikz J, et al. Management of acute whiplash: a randomized controlled trial of multidisciplinary stratified treatments. Pain 2013;154:1798-806.

48. Jagnoor J, Cameron I, Harvey L, et alMotor accidents authority: guidelines for the management of acute whiplash-associated disorders-for health professionals. 3rd edn. Sydney: NSW Government, 2014.

49. Tough EA, White AR, Richards SH, et al. Myofascial trigger point needling for whiplash associated pain-a feasibility study. Man Ther 2010;15:529-35.

50. Rushton A, Rivett D, Carlesso L, et al. International framework for examination of the cervical region for potential of cervical arterial dysfunction prior to orthopaedic manual therapy intervention. Man Ther 2014;19:222-8.

51. Crawford JR, Khan RJK, Varley GW. Early management and outcome following soft tissue injuries of the neck-a randomised controlled trial. Injury 2004;35:891-5.

52. Rosenfeld M, Gunnarsson R, Borenstein P. Early intervention in whiplash-associated disorders: a comparison of two treatment protocols. Spine (Phila Pa 1976) 2000;25:1782-7.

53. Rosenfeld M, Seferiadis A, Carlsson J, et al. Active intervention in patients with whiplash-associated disorders improves long-term prognosis: a randomized controlled clinical trial. Spine (Phila Pa 1976) 2003;28:2491-8.

54. Richter M, Ferrari R, Otte D, et al. Correlation of clinical findings, collision parameters, and psychological factors in the outcome of whiplash associated disorders. J Neurol Neurosurg Psychiatr 2004;75:758-64

55. Lamb SE, Gates S, Underwood MR, et al. Managing Injuries of the Neck Trial (MINT): design of a randomised controlled trial of treatments for whiplash associated disorders. BMC Musculoskelet Disord 2007;8:7.

56. Hoffmann TC, Glasziou PP, Boutron I, et al. Better reporting of interventions: template for intervention description and replication (TIDieR) checklist and guide. BMJ 2014;348:g1687.

57. Thomeé P. Self-efficacy of knee function in patients with an anterio cruciate ligament injury. Institute of Clincial Sciences. Department of Orthopaedics, 2007.

58. Börsbo B, Gerdle B, Peolsson M. Impact of the interaction between self-efficacy, symptoms and catastrophising on disability, quality of life and health in with chronic pain patients. Disabil Rehabil 2010;32:1387-96.

59. Barlow J. Self-efficacy in the context of rehabilitation. International Encyclopedia of Rehabilitaition. 2013

60. Vernon $\mathrm{H}$, Mior $\mathrm{S}$. The neck disability index: a study of reliability and validity. J Manipulative Physiol Ther 1991;14:409-15.

61. Pietrobon R, Coeytaux RR, Carey TS, et al. Standard scales for measurement of functional outcome for cervical pain or dysfunction: a systematic review. Spine (Phila Pa 1976) 2002;27:515-22.

62. Vernon $\mathrm{H}$. The neck disability index: state-of-the-art, 1991-2008. $J$ Manipulative Physiol Ther 2008;31:491-502.

63. MacDermid JC, Walton DM, Avery S, et al. Measurement properties of the neck disability index: a systematic review. J Orthop Sports Phys Ther 2009;39:400-17.

64. Walton DM, MacDermid JC, Giorgianni AA, et al. Risk factors for persistent problems following acute whiplash injury: update of a systematic review and meta-analysis. J Orthop Sports Phys Ther 2013:43:31-43.

65. Lamb SE, Gates S, Williams MA, et al. Emergency department treatments and physiotherapy for acute whiplash: a pragmatic, two-step, randomised controlled trial. Lancet 2013;381:546-56.

66. Huskisson E. Measurement of pain. Lancet 1974;304:1127-31.

67. Bijur PE, Silver W, Gallagher EJ. Reliability of the visual analog scale for measurement of acute pain. Acad Emerg Med 2001:8:1153-7.

68. Myles PS, Urquhart N. The linearity of the visual analogue scale in patients with severe acute pain. Anaesth Intensive Care 2005;33:54-8.

69. Price DD, Patel R, Robinson ME, et al. Characteristics of electronic visual analogue and numerical scales for ratings of experimental pain in healthy subjects and fibromyalgia patients. Pain 2008; $140: 158-66$.

70. Hendriks EJ, Scholten-Peeters GG, van der Windt DA, et al. Prognostic factors for poor recovery in acute whiplash patients. Pain 2005;114:408-16.

71. Stovner LJ. The nosologic status of the whiplash syndrome: a critical review based on a methodological approach. Spine (Phila Pa 1976) 1996:21:2735-46.

72. Dall'Alba PT, Sterling MM, Treleaven JM, et al. Cervical range of motion discriminates between asymptomatic persons and those with whiplash. Spine (Phila Pa 1976) 2001;26:2090-4.

73. Kasch H, Bach FW, Jensen TS. Handicap after acute whiplash injury: a 1-year prospective study of risk factors. Neurology 2001:56:1637-43.

74. Hole DE, Cook JM, Bolton JE. Reliability and concurrent validity of two instruments for measuring cervical range of motion: effects of age and gender. Man Ther 1995;1:36-42.

75. Malmström EM, Karlberg M, Melander A, et al. Zebris versus Myrin: a comparative study between a three-dimensional ultrasound movement analysis and an inclinometer/compass method: intradevice reliability, concurrent validity, intertester comparison, intratester reliability, and intraindividual variability. Spine (Phila Pa 1976) 2003;28:E433-40.

76. Williams MA, McCarthy CJ, Chorti A, et al. A systematic review of reliability and validity studies of methods for measuring active and passive cervical range of motion. J Manipulative Physiol Ther 2010;33:138-55.

77. Williams MA, Williamson E, Gates S, et al. Reproducibility of the cervical range of motion (CROM) device for individuals with sub-acute whiplash associated disorders. Eur Spine $J$ 2012;21:872-8. 
78. Vanderweeën L, Oostendorp RA, Vaes $\mathrm{P}$, et al. Pressure algometry in manual therapy. Man Ther 1996;1:258-65.

79. Sterling M, Jull G, Vicenzino B, et al. Sensory hypersensitivity occurs soon after whiplash injury and is associated with poor recovery. Pain 2003;104:509-17.

80. Sterling M, Jull G, Vicenzino B, et al. Characterization of acute whiplash-associated disorders. Spine (Phila Pa 1976) 2004;29:182-8.

81. Fernández-Pérez AM, Villaverde-Gutiérrez C, Mora-Sanchez A, et al. Muscle trigger points, pressure pain threshold, and cervical range of motion in patients with high level of disability related to acute whiplash injury. J Orthop Sports Phys Ther 2012;42: 634-41.

82. Stone AM, Vicenzino B, Lim EC, et al. Measures of central hyperexcitability in chronic whiplash associated disorder-a systematic review and meta-analysis. Man Ther 2013;18:111-17.

83. Carroll LJ, Hurwitz EL, Côté $P$, et al. Research priorities and methodological implications: the bone and joint decade 2000-2010 Task Force on neck pain and its associated disorders. Spine (Phila Pa 1976) 2008;33(Suppl 4):S214-20.

84. Kinser AM, Sands WA, Stone MH. Reliability and validity of a pressure algometer. J Strength Cond Res 2009;23:312-14.

85. Walton DM, Macdermid JC, Nielson W, et al. Reliability, standard error, and minimum detectable change of clinical pressure pain threshold testing in people with and without acute neck pain. $J$ Orthop Sports Phys Ther 2011:41:644-50.

86. Kardouni JR, Shaffer SW, Pidcoe PE, et al. Immediate changes in pressure pain sensitivity after thoracic spinal manipulative therapy in patients with subacromial impingement syndrome: a randomized controlled study. Man Ther 2015;20:540-6.

87. Walton DM, Levesque L, Payne M, et al. Clinical pressure pain threshold testing in neck pain: comparing protocols, responsiveness, and association with psychological variables. Phys Ther 2014:94:827-37.

88. Horowitz M, Wilner N, Alvarez W. Impact of event scale: a measure of subjective stress. Psychosom Med 1979;41:209-18.

89. Zilberg NJ, Weiss DS, Horowitz MJ. Impact of event scale: a cross-validation study and some empirical evidence supporting a conceptual model of stress response syndromes. J Consult Clin Psychol 1982;50:407-14

90. Sundin EC, Horowitz MJ. Impact of event scale: psychometric properties. Br J Psychiatry 2002;180:205-9.

91. Sterling M, Jull G, Vicenzino B, et al. Physical and psychological factors predict outcome following whiplash injury. Pain 2005;114:141-8.
92. Buitenhuis J, de Jong PJ, Jaspers JP, et al. Relationship between posttraumatic stress disorder symptoms and the course of whiplash complaints. J Psychosom Res 2006;61:681-9.

93. Asmundson GJ, Katz J. Understanding pain and posttraumatic stress disorder comorbidity: do pathological responses to trauma alter the perception of pain? Pain 2008;138:247-9.

94. Pedler A, Sterling M. Assessing fear-avoidance beliefs in patients with whiplash-associated disorders: a comparison of 2 measures. Clin J Pain 2011;27:502-7.

95. Vernon $\mathrm{H}$, Guerriero R, Soave D, et al. The relationship between self-rated disability, fear-avoidance beliefs, and nonorganic signs in patients with chronic whiplash-associated disorder. J Manipulative Physiol Ther 2011;34:506-13.

96. Kamper SJ, Maher CG, Menezes Costa Lda C, et al. Does fear of movement mediate the relationship between pain intensity and disability in patients following whiplash injury? A prospective longitudinal study. Pain 2012;153:113-19.

97. Buitenhuis J, de Jong PJ. Fear avoidance and illness beliefs in post-traumatic neck pain. Spine (Phila Pa 1976) 2011;36(Suppl 25):S238-43.

98. Cleland JA, Fritz JM, Childs JD. Psychometric properties of the Fear-Avoidance Beliefs Questionnaire and Tampa Scale of Kinesiophobia in patients with neck pain. Am J Phys Med Rehabil 2008;87:109-17.

99. Hallal PC, Simoes E, Reichert FF, et al. Validity and reliability of the telephone-administered international physical activity questionnaire in Brazil. J Phys Act Health 2010;7:402-9.

100. Brooks R, Group E. EuroQol: the current state of play. Health Policy 1996;37:53-72.

101. Hertzog MA. Considerations in determining sample size for pilot studies. Res Nurs Health 2008;31:180-91.

102. Hsieh HF, Shannon SE. Three approaches to qualitative content analysis. Qual Health Res 2005;15:1277-88.

103. Corbin J, Strauss A. Grounded theory research: procedures, canons, and evaluative criteria. Qual Sociol 1990;13:3-21.

104. Kitzinger J. Qualitative research. Introducing focus groups. BMJ 1995;311:299.

105. Bos C, Van der Lans IA, Van Rijnsoever FJ, et al. Understanding consumer acceptance of intervention strategies for healthy food choices: a qualitative study. BMC Public Health 2013;13:1073.

106. King J, Anderson CM. Patient safety and physiotherapy: what does it mean for your clinical practice? Physiother Can 2010;62:172-9. 